\title{
Application of adjustment calculus in the nodeless Trefftz method for a problem of two-dimensional temperature field of the boiling liquid flowing in a minichannel
}

\author{
Sylwia Hożejowska ${ }^{1, a}$, Beata Maciejewska ${ }^{1}$ and Leszek Hożejowski ${ }^{1}$ \\ ${ }^{1}$ Kielce University of Technology, AL. 1000-lecia PP 7, 25-314 Kielce Poland
}

\begin{abstract}
The paper presents application of the nodeless Trefftz method to calculate temperature of the heating foil and the insulating glass pane during continuous flow of a refrigerant along a vertical minichannel. Numerical computations refer to an experiment in which the refrigerant (FC-72) enters under controlled pressure and temperature a rectangular minichannel. Initially its temperature is below the boiling point. During the flow it is heated by a heating foil. The thermosensitive liquid crystals allow to obtain twodimensional temperature field in the foil. Since the nodeless Trefftz method has very good performance for providing solutions to such problems, it was chosen as a numerical method to approximate two-dimensional temperature distribution in the protecting glass and the heating foil. Due to known temperature of the refrigerant it was also possible to evaluate the heat transfer coefficient at the foil-refrigerant interface. For expected improvement of the numerical results the nodeless Trefftz method was combined with adjustment calculus. Adjustment calculus allowed to smooth the measurements and to decrease the measurement errors. As in the case of the measurement errors, the error of the heat transfer coefficient decreased.
\end{abstract}

\section{Introduction}

Progress in technology leads to miniaturization of technical devices which can be downsized without loss of their functionality. This trend is followed by engineers' efforts to construct miniature heat exchangers which, due to phase change of a refrigerant, can be employed for efficient high heat flux removal at low temperature difference between the refrigerant and the surface to be cooled.

\section{Experimental research}

The experiment referring to the considered problem, presented and discussed in detail in [1-3], will be described shortly in this paper. The main element of experimental stand is a module with a vertical minichannel of rectangular cross section, designed for flow experiments with FC-72 fluid. One of the walls of the minichannel is a heating foil made of Haynes-230 superalloy with uniformly distributed micro-holes machined by laser technology. DC power supplied to the foil can be regulated. The liquid crystal film which is spread over the heating foil allows to obtain visualization of two-dimensional temperature distribution of the surface. The heating foil is separated from ambient space by a protecting glass barrier. Due to the other glass pane isolating the minichannel from the opposite side, one can observe flow structures and void fraction. The liquid (FC-72) whose temperature is below saturation temperature and whose pressure is known (and controlled), flows in the minichannel where it is heated by the foil at temperature exceeding the saturation temperature. Measurements of temperature and pressure of the two phase liquid are made at the minichannel outlet. The other measured parameter concerning the flow is mass flux and those concerning the power supply are output voltage and current. Collecting measurement data begins when the process is in a steady state.

\section{Mathematical model}

For simplicity, we assume two-dimensional flow conditions, neglecting temperature and velocity variation along the width of the minichannel. Our considerations focused on the central part of the measurement module (along its height) so that the physical phenomena on the side edges did not affect thermodynamic parameters within the investigated segment.

To use a clear notation in further mathematical equations, the subscripts $G, F, f$ refer to protecting glass, heating foil and fluid, respectively. It is assumed that stationary temperature distributions in the protecting

\footnotetext{
${ }^{\text {a }}$ Corresponding author: ztpsf@tu.kielce.pl
} 
glass and heating foil are described, as in $[1,4,5]$, by Laplace and Poisson equation, respectively:

a) for glass

$$
\nabla^{2} T_{G}=0 \text { for }(x, y) \in \Omega_{G}
$$

where

$$
\Omega_{G}=\left\{(x, y) \in R^{2}: 0<x<L, \quad 0<y<\delta_{G}\right\}
$$

b) for foil

$$
\nabla^{2} T_{F}=-\frac{q_{V}}{\lambda_{F}} \text { for }(x, y) \in \Omega_{F}
$$

where

$$
\Omega_{F}=\left\{(x, y) \in R^{2}: x_{1}<x<x_{P}, \quad \delta_{G}<y<\delta_{G}+\delta_{F}\right\}
$$

The symbols used in formulas (1) - (4) mean: $\nabla^{2}=\frac{\partial^{2}}{\partial x^{2}}+\frac{\partial^{2}}{\partial y^{2}}, \quad L-$ minichannel height, $q_{V}-$ volumetric heat flux, $\lambda$ - thermal conductivity, $x_{1}$ and $x_{P}-x$-coordinate of the $n$-th measurement point where $n=1$ and $n=P$, respectively, $P-$ the number of measurement points.

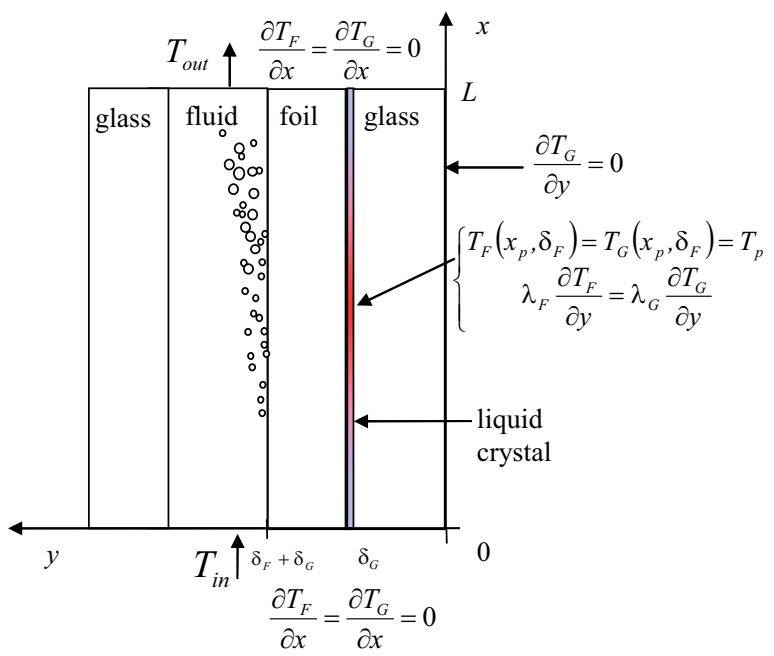

Figure 1. Measurement module and boundary conditions. (Picture not scaled proportionally).

We assume a perfect thermal contact between the foil and the glass pane, which gives

$$
\begin{gathered}
T_{F}\left(x_{p}, \delta_{G}\right)=T_{G}\left(x_{p}, \delta_{G}\right)=T_{p} \text { for } p=1,2, \ldots, P \\
T_{F}\left(x, \delta_{G}\right)=T_{G}\left(x, \delta_{G}\right) \text { for } 0 \leq x \leq L \\
\lambda_{F} \frac{\partial T_{F}}{\partial y}=\lambda_{G} \frac{\partial T_{G}}{\partial y} \text { for } y=\delta_{G}, 0 \leq x \leq L
\end{gathered}
$$

where $T_{p}$ denotes temperature measurement at the $p$-th point.
The remaining boundaries of the foil and glass are insulated which is indicated in figure 1 by proper equations.

\section{The nodeless Trefftz method}

The two-dimensional temperature distribution in glass and in foil was computed by Trefftz method, both classic and nodeless. According to the concept of Trefftz method, the unknown solution to a governing differential equation is approximated with a linear combination of Trefftz functions satisfying the governing equation. Trefftz functions suitable for equations (1) and (3) are harmonic polynomials. Temperature measurements obtained by liquid crystal thermography and required for numerical computations were smoothed by Trefftz functions, [4]. To determine semi-analytical solution for two-dimensional temperature distribution in the glass, $T_{G}$, one can use Trefftz method following a computational procedure described in detail in [5]. Approximate temperature distribution $T_{F}$, in the part (4) of the heating foil can be obtained by discontinuous Trefftz method (the nodeless Trefftz method). In order to solve for $T_{F}$, the region $\Omega_{F}$ will be divided into $J$ subregions

$$
\begin{gathered}
\Omega_{F}^{j}=\left\{(x, y) \in R^{2}: x_{j} \leq x \leq x_{j+1}, \quad \delta_{G} \leq y \leq \delta_{G}+\delta_{F}\right\} \\
\text { for } j=1,2, \ldots, J
\end{gathered}
$$

In each of the subregions $\Omega_{F}^{j}$ the temperature of the heating foil $T_{F}^{j}$, is approximated with a formula containing a linear combination of Trefftz functions

$$
T_{F}^{j}(x, y)=u(x, y)+\sum_{i=1}^{M} a_{i j} v_{i}(x, y)
$$

where $u(x, y)$ denotes a particular solution to (3) and $v_{i}(x, y)$ are Trefftz functions (harmonic polynomials) for Laplace equation, $M$ - the number of Trefftz functions. The coefficients $a_{i j}$ in formula (9) are calculated from the minimum of a functional $H_{F}$

$$
\begin{aligned}
& H_{F}=\int_{\delta_{G}}^{\sigma_{G}+\delta_{F}}\left(T_{F}^{1}\left(x_{1}, y\right)-T_{1}\right)^{2} d y+ \\
& +\int_{\delta_{G}}^{\delta_{G}+\delta_{F}}\left(T_{F}^{K}\left(x_{P}, y\right)-T_{P}\right)^{2} d y+ \\
& +\sum_{p_{j}=1}^{P}\left(T_{F}^{j}\left(x_{p_{j}}, \delta_{G}\right)-T_{p_{j}}\right)^{2}+ \\
& +\sum_{j=1}^{K} \int_{x_{j}}^{\sigma_{j+1}}\left(T_{F}^{j}\left(x, \delta_{G}\right)-T_{G}\left(x, \delta_{G}\right)\right)^{2} d x+ \\
& +\sum_{j=1}^{K} \int_{x_{j}}^{\sigma_{j+1}}\left(\lambda_{F} \frac{\partial T_{F}^{j}}{\partial y}\left(x, \delta_{G}\right)-\lambda_{G} \frac{\partial T_{G}}{\partial y}\left(x, \delta_{G}\right)\right)^{2} d x+ \\
& +\sum_{j=1}^{K-1} \int_{\delta_{G}}^{\delta_{G}+\delta_{F}}\left(T_{F}^{j}\left(x_{j+1}, y\right)-T_{F}^{j+1}\left(x_{j+1}, y\right)\right)^{2} d y+ \\
& +\sum_{j=1}^{K-1} \int_{\delta_{G}}^{\delta_{G}+\delta_{F}}\left(\frac{\partial T_{F}^{j}}{\partial x}\left(x_{j+1}, y\right)-\frac{\partial T_{F}^{j+1}}{\partial x}\left(x_{j+1}, y\right)\right)^{2} d y
\end{aligned}
$$


The first terms of the functional (10) represent average squared error between computed and prescribed values of the heating foil temperature and heat flux in every subregion $\Omega_{F}^{j}$ while the remaining terms are responsible for good agreement between temperature and its gradient along the common boundaries of the subregions $\Omega_{F}^{j}$. The obtained approximate temperatures $T_{G}$ and $T_{F}$ satisfy the differential equations (1) and (3), respectively, but the boundary conditions are fulfilled by the approximants $T_{G}$ and $T_{F}$ only in variational sense.

Having calculated temperature distribution in the heating foil in every subregion $\Omega_{F}^{j}$, one can easily determine the heat transfer coefficient from the formula

$$
\alpha^{j}(x)=\frac{-\lambda_{F} \frac{\partial T_{F}^{j}\left(x, \delta_{G}+\delta_{F}\right)}{\partial y}}{T_{F}^{j}\left(x, \delta_{G}+\delta_{F}\right)-T_{f}(x)}
$$

where fluid temperature $T_{f}(x)$ is approximated linearly from the value $T_{i n}$ at the channel inlet to the value $T_{\text {out }}$ at the channel outlet

$$
T_{f}(x)=T_{\text {in }}+\frac{T_{\text {out }}-T_{\text {in }}}{L} x
$$

\subsection{Adjustment calculus}

For the sake of accuracy in determining two-dimensional temperature distribution in the glass and foil, temperature measurements will be approximated with Trefftz functions as follows

$$
T_{p} \approx T\left(x_{p}, \delta_{G}\right)=\sum_{i=1}^{I} b_{i} v_{i}\left(x_{p}, \delta_{G}\right)
$$

Trefftz functions in eq. (13) are selected so that the columns of the matrix

$$
V_{p i}=v_{i}\left(x_{p}, \delta_{G}\right)
$$

be linearly independent. Estimation according to eq. (13) assumes that the temperature measurements are biased. It seems to be more realistic that the temperature measurements $T_{p}$ suffer from random measurement errors. In fact, in our experiment we obtain $P$ measurement values; each can be expressed by two parts - a ,true" temperature and an error which is unsystematic (random) and different for different measurements. The following formula allows to express it in terms of mathematical equations

$$
\sum_{i=1}^{I} b_{i} v_{i}\left(x_{p}, \delta_{G}\right)-T_{p}=\Delta_{p} \neq 0
$$

Temperature measurements $T_{p}$ approximated by the Trefftz functions (13) can be corrected by adjustment calculus as shown in $[4,6]$. In such an approach we replace measurements $T_{p}$ with "new" (corrected) measurements $\widetilde{T}_{p}$, demanding that the corrections $\varepsilon_{p}$ contained in the formula

$$
\widetilde{T}_{p}=T_{p}+\varepsilon_{p}
$$

have a normal distribution with expected value equal to zero and a finite variance $\sigma_{p}^{2}$, [6]. The $\sigma_{p}$ value reflects the error of heating foil temperature estimation based on the hue indicated by liquid crystals, [5]. Corrections $\varepsilon_{p}$ are determined so as to minimize Lagrange'a function

$$
\Phi=\sum_{p=1}^{P}\left(\frac{\varepsilon_{p}}{\sigma_{p}}\right)^{2}+2 \sum_{p=1}^{P} \omega_{k}\left(\widetilde{T}_{p}-T\left(x_{p}, \delta_{G}\right)\right) \rightarrow \min
$$

where $\omega_{p}$ - Lagrange multipliers.

Having corrected temperature measurements, we recalculated measurement errors $\sigma_{p}^{\text {corr }}$ according to the error propagation law, [4,6]. With known values of $\widetilde{T}_{p}$ it was possible to recalculate approximate temperatures of the protecting glass, $\widetilde{T}_{G}$, and the heating foil, $\widetilde{T}_{F}$, as described in [4].

\section{Results}

Numerical calculations were performed for the data coming from the experiments described in [1-3] and related to the forced flow of FC-72 through an asymmetrically heated minichannel, see figure 2 .

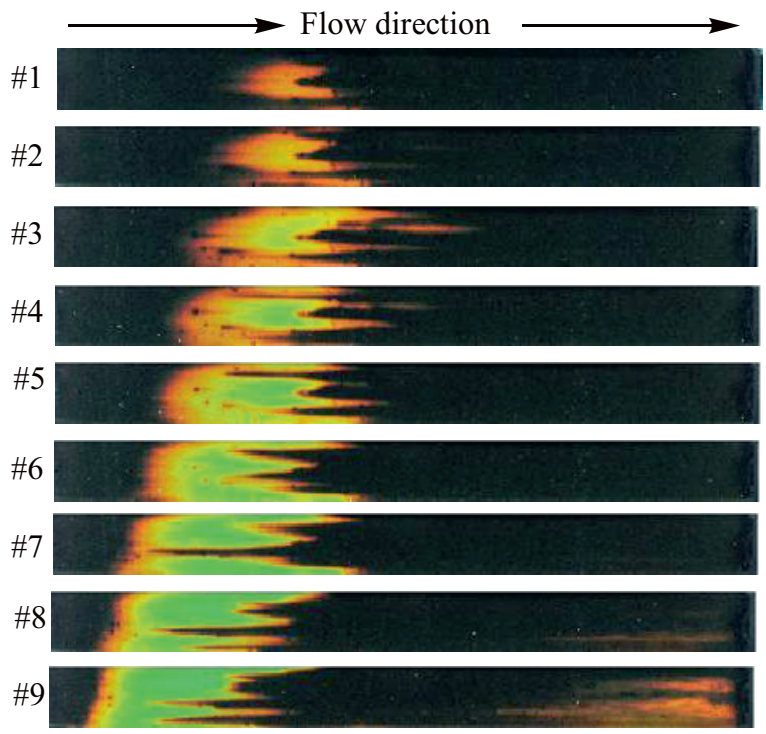

Figure 2. Hue distribution on the minichannel external surface while increasing the heat flux supplied to the heating foil. Experimental parameters of the runs: mass flux $285 \mathrm{~kg} /\left(\mathrm{m}^{2} \mathrm{~s}\right)$; $\mathrm{Re}=884$; inlet presser $124 \mathrm{kPa}$, volumetric heat flux $7.96 \cdot 10^{4} \div 1.24 \cdot 10^{5} \mathrm{~kW} / \mathrm{m}^{3}$; foil parameters: $\delta_{F}=1.02 \cdot 10^{-4} \mathrm{~m}$, $L=0.3 \mathrm{~m}, \lambda_{F}=8.3 \mathrm{~W} /(\mathrm{mK})$; glass parameters: $\delta_{G}=0.005 \mathrm{~m}$, $\lambda_{G}=0.71 \mathrm{~W} /(\mathrm{mK})$.

Measured values included temperature of FC-72 at the inlet and outlet of the minichannel, flow velocity, 
pressure at the minichannel inlet and outlet, voltage drop and electrical current supplied to the foil. Liquid crystal thermography helped calculate approximate temperature of the heating foil in contact with the protecting glass.

Approximate temperature of the glass was computed with a use of 16 Trefftz functions. The same number of Trefftz functions were used for approximation of temperature of the heating foil in the region $\Omega_{F}$ which, for accurate results, required division into 4 subregions according to (4). Computations were performed for the data smoothed by adjustment calculus in the manner described in paragraph 3.1.

Figure 3 presents two-dimensional distribution of glass and heating foil temperature calculated with nodeless Trefftz method for the data smoothed by adjustment calculus.

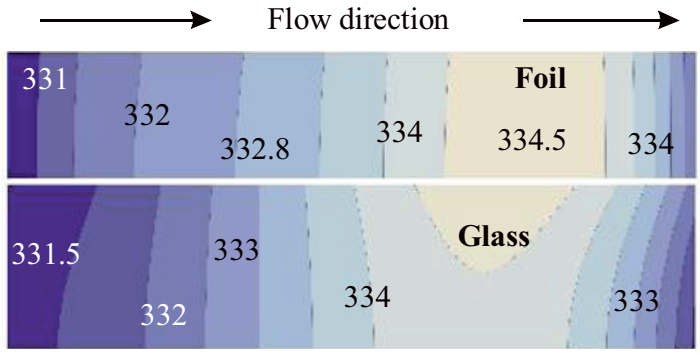

Figure 3. Temperature of the protecting glass by Trefftz method and temperature of the heating foil by nodeless Trefftz method (both after applying adjustment calculus). Setting \#3.
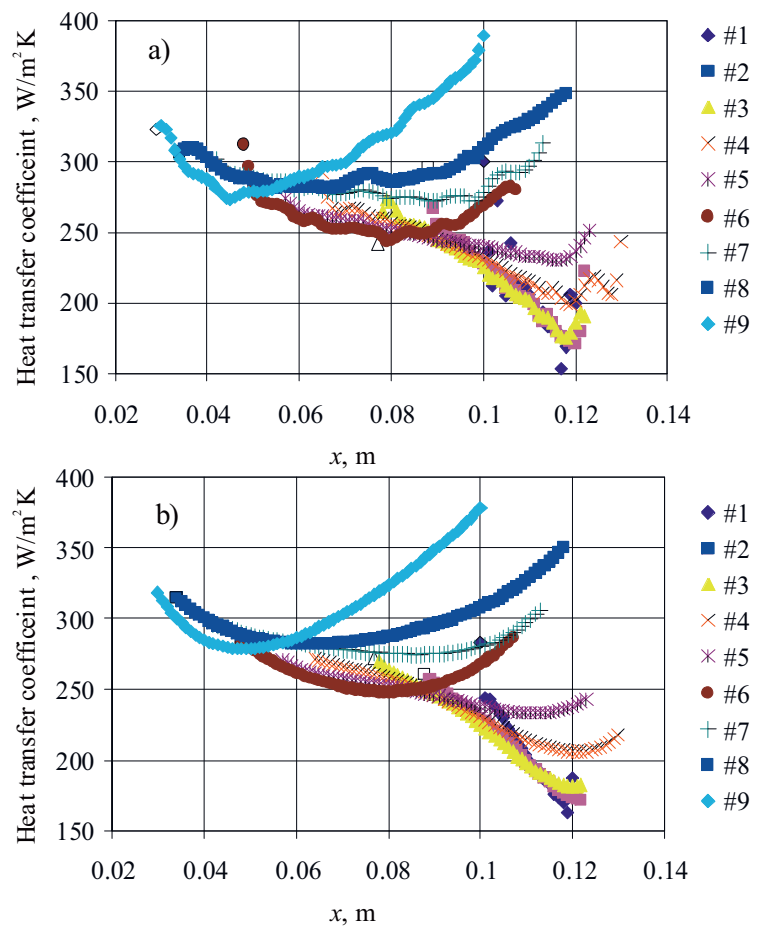

Figure 4. Heat transfer coefficient as a function of the minichannel height obtained by: a) nodeless Trefftz method, b) nodelees Trefftz method after applying adjustment calculus.
The figure 4 shows the heat transfer coefficient as a function of the minichannel height, calculated from (11) for the data presented in figure 2 .

The mean error in estimation of the heat transfer coefficient (see [3]) for settings \#1 to \#9 turned to be equal to $5.4 \%$. After application of adjustment calculus the error decreased to $3.5 \%$.

In [7] were proposed mathematical models describing heat transfer in three areas (protecting glass, heating foil and fluid). Trefftz method was used to determine the twodimensional temperature distribution also in the liquid in bubbly and bubbly-slug flow. The computations were based on temperature measurements of foil obtained by liquid crystal thermography and experimentally calculated void fraction.

\section{Conclusions}

1. Trefftz method and nodeless Trefftz method were applied for solving a direct problem of determining the temperature distribution in the protecting glass and also for solving an inverse problem of determining the temperature distribution in the heating foil.

2. Trefftz method (as well as nodeless Trefftz method) can be combined with adjustment calculus. In the considered problem both gave satisfying results.

3. Measurement data approximation with Trefftz functions helps to smooth the measurements and decreases measurement errors.

4. When calculated from measurements corrected by adjustment calculus, the heat transfer coefficient has significantly smaller error in comparison with that calculated without adjustment calculus.

\section{References}

1. M. Piasecka, B. Maciejewska, B., Exp Therm Fluid SCI, 38 19-32 (2012)

2. M.Piasecka, Int. J. Heat and Mass Transf., 66472 488 (2013)

3. M.Piasecka., Heat Mass Transf. 49 261-271 (2013)

4. K.Grysa, S.Hożejowska, B.Maciejewska, JTAM, 50 1087-1096 (2012)

5. S. Hozejowska, M. Piasecka, M.E. Poniewski, Int. J. Therm. Sci., 48 1049-1059 (2009)

6. S.Brandt, Data analysis, statistical and computational methods for scientists and engineers, (New York, Springer Verlag, 1999)

7. S.Hożejowska, R. Kaniowski, M.E. Poniewski, , Int. J. Numerical Methods for Heat and Fluid Flow, (to be published) 